\title{
REVIEW
}

\section{Defining the Epidemiology and Burden of Severe Respiratory Syncytial Virus Infection Among Infants and Children in Western Countries}

\author{
Louis Bont · Paul A. Checchia · Brigitte Fauroux · Josep Figueras-Aloy • \\ Paolo Manzoni · Bosco Paes · Eric A. F. Simões · Xavier Carbonell-Estrany
}

Received: June 15, 2016 / Published online: August 1, 2016

(C) The Author(s) 2016. This article is published with open access at Springerlink.com

\begin{abstract}
Introduction: The REGAL (RSV [respiratory syncytial virus] Evidence-a Geographical Archive of the Literature) series provides a comprehensive review of the published evidence in the field of RSV in Western countries over the last 20 years. This first of
\end{abstract}

Enhanced content To view enhanced content for this article go to http://www.medengine.com/Redeem/ F6E4F06044E2A44B.

Electronic supplementary material The online version of this article (doi:10.1007/s40121-016-0123-0) contains supplementary material, which is available to authorized users.

L. Bont

University Medical Center Utrecht, Utrecht, The Netherlands

\section{P. A. Checchia}

Baylor College of Medicine, Texas Children's

Hospital Houston, Texas, USA

B. Fauroux

Necker University Hospital and Paris 5 University,

Paris, France

\section{J. Figueras-Aloy}

Hospital Clínic, Catedràtic de Pediatria, Universitat de Barcelona, Barcelona, Spain

P. Manzoni

Neonatology and NICU, Sant'Anna Hospital, Turin, Italy seven publications covers the epidemiology and burden of RSV infection.

Methods: A systematic review was undertaken for articles published between Jan 1, 1995 and Dec 31, 2015 across PubMed, Embase, The Cochrane Library, and Clinicaltrials.gov. Studies reporting data for hospital visits/admissions for RSV infection among children ( $\leq 18$ years of age), as well as studies reporting RSV-associated morbidity, mortality, and risk factors were included. Study quality and strength of evidence (SOE) were graded using recognized criteria.

Result: 2315 studies were identified of which 98 were included. RSV was associated with

B. Paes

Department of Pediatrics (Neonatal Division), McMaster University, Hamilton, ON, Canada

\section{E. A. F. Simões}

Colorado School of Public Health, University of

Colorado School of Medicine, Aurora, CO, USA

X. Carbonell-Estrany $(\bowtie)$

Hospital Clinic, Institut d'Investigacions

Biomediques August Pi Suñer (IDIBAPS), Barcelona, Spain

e-mail: carbonell@comb.cat 
$12-63 \%$ of all acute respiratory infections (ARIs) and $19-81 \%$ of all viral ARIs causing hospitalizations in children (high SOE). Annual RSV hospitalization (RSVH) rates increased with decreasing age and varied by a factor of 2-3 across seasons (high SOE). Studies were conflicting on whether the incidence of RSVH has increased, decreased, or remained stable over the last 20 years (moderate SOE). Length of hospital stay ranged from 2 to 11 days, with $2-12 \%$ of cases requiring intensive care unit admission (moderate SOE). Case-fatality rates were $<0.5 \%$ (moderate SOE). Risk factors associated with RSVH included: male sex; age $<6$ months; birth during the first half of the RSV season; crowding/siblings; and day-care exposure (high SOE).

Conclusion: RSV infection remains a major burden on Western healthcare systems and has been associated with significant morbidity. Further studies focusing on the epidemiology of RSV infection (particularly in the outpatient setting), the impact of co-infection, better estimates of case-fatality rates and associated risk factors (all currently moderate/low SOE) are needed to determine the true burden of disease.

Funding: Abbvie.

Keywords: Acute respiratory infection; Bronchiolitis; Burden; Epidemiology; Lower respiratory tract infection; Respiratory syncytial virus

\section{INTRODUCTION}

Respiratory syncytial virus (RSV) is the most common cause of infection of the upper and lower respiratory tract in infants and young children worldwide and a major public health burden [1-10]. Globally, there are an estimated 33 million annual cases of RSV-associated lower respiratory tract infection in children under the age of 5 years, with at least 3.4 million episodes necessitating admission to hospital [1]. Although the risk of hospital admission is higher in known risk groups, such as those born prematurely, in particular, those with chronic lung disease (CLD)/bronchopulmonary dysplasia (BPD), and those with congenital heart disease (CHD) [11-15], the majority of severe cases of RSV infection occur among previously healthy term infants $[14,16]$. Severe RSV infection remains a significant unmet medical need, and reducing the health burden of RSV has become a priority of the World Health Organization's (WHO) BRaVe (Battle against Respiratory Viruses) initiative $[17,18]$. Part of the research agenda of this initiative is to define the burden of respiratory viral infections [18].

Over the last 20 years, there has been a considerable body of research published on RSV that has improved our understanding of the epidemiology in different risk groups, the acute and long-term burden to children and to healthcare systems, the underlying infection process and genetics, as well as preventive and management strategies. An understanding of the incidence and complications of RSV disease is essential for planning strategies to control RSV infection and to optimize the use of RSV prophylaxis and future RSV vaccines [19-21].

To provide a comprehensive understanding on severe RSV disease in infants and children in Western societies, an expert panel, comprising Neonatologists, Pediatricians, Pediatric Infectious Disease Specialists, Pediatric Cardiologists and Pediatric Pulmonologists from the United States, Canada and Europe, undertook an evidence-based search of the literature which has accumulated over the past two decades. The primary objective of REGAL (RSV Evidence-a Geographical Archive of the Literature) was to carry out a series of systematic 
literature reviews and, then, to assess, quantify, summarize and grade the evidence base. By undertaking this review, the current state of the art in our understanding of RSV was defined as well as, importantly, gaps in our knowledge and future areas of research.

This paper represents the first of a series of seven publications encompassing different areas of the systematic review and covers in detail the overall methodology that was followed for each of the reviews, and summarizes the first topic: the epidemiology and burden of severe RSV infection requiring hospitalization among infants and children in Western societies.

\section{METHODS}

\section{Objectives}

The primary objective of REGAL was to address seven specific research questions (Table 1). The systematic literature reviews undertaken to answer each of these research questions all used the same broad methodology, which is described in detail below.

\section{Search Strategy and Selection Criteria}

Following a study protocol with pre-defined search terms, we conducted a systematic and comprehensive search of the medical literature electronically indexed in MEDLINE (PubMed), Embase and The Cochrane Library. Search strategies were devised for each systematic review and combined free-text search terms with medical subject headings (MeSH). An important part of the protocol was the countries to be included. The overall burden of RSV has been studied in many industrialized countries, and therefore, to ensure a manageable volume of publications, only studies conducted in Western countries were included, which we defined as the United States, Canada, and Europe (including Turkey and the Russian

Table 1 REGAL: study questions

1. What is the overall epidemiology and disease burden of severe RSV infection in Western countries, and what are the associated risk factors for RSVH?

2. What is the predisposition and associated morbidity, long-term sequelae and mortality of preterm infants $(<37 \mathrm{wGA})$ without CLD/BPD or CHD, overall, and split by gestational age segments, to severe RSV infection, and what are the risk factors associated with RSVH?

3. What is the predisposition and associated morbidity, long-term sequelae and mortality of infants with underlying CLD/BPD to severe RSV infection in Western countries?

4. What is the predisposition and associated morbidity, long-term sequelae and mortality of infants with underlying CHD to severe RSV infection in Western countries?

5. What is the nature, incidence and impact of long-term respiratory morbidity associated with RSVH in infancy in Western countries, specifically early and late wheeze?

6. What other groups of infants with underlying medical conditions or chronic diseases are at high risk of RSVH and associated morbidity?

7. What are the optimal approaches and strategies for the prevention and treatment of severe RSV infection and what are the future perspectives in this regard?

$B P D$ bronchopulmonary dysplasia, $C H D$ congenital heart disease, $C L D$ chronic lung disease, $R S V$ respiratory syncytial virus, $R S V H$ RSV hospitalization, $w G A$ weeks' gestational age 
Federation). The full protocol for the systematic reviews is available as part of the online supplement (Supplementary Material 1REGAL Protocol). Our search included studies conducted in children (defined as $\leq 18$ years) and published between January 1, 1995 and December 31, 2015. The target populations for REGAL included previously healthy term or preterm children $[<37$ week gestational age (wGA)], those with CLD/BPD, CHD, or other high-risk comorbid conditions (e.g., anatomic pulmonary abnormalities, neuromuscular disorders, Down syndrome, immunodeficiencies and cystic fibrosis) with 'proven' or 'probable' RSV. Children who had received immunoprophylaxis with palivizumab were included.

No language limits were set on the database searches, with the caveat that English translations of at least the abstract had to be available. Since there is not a universal, standardized definition of severe RSV disease, for the purposes of this article, we have taken this to be 'RSV infection requiring hospitalization'. Randomized controlled trials, non-randomized controlled trials, crossover trials, single-arm studies, cohort studies (prospective and retrospective), case-control studies (prospective and retrospective), and case series were included, as well as published data from registries and medical databases. We also searched the database ClinicalTrials.gov, which provides information on current ongoing clinical research studies being conducted around the world. In addition, we hand-searched online journals and scanned the reference lists of identified citations and relevant abstracts presented at key meetings to find additional relevant publications for each of the reviews.

Two reviewers (J. Smith and J. Blake) undertook the search adopting a two-phase screening process. In Phase 1, the title (Stage 1) and, then, the abstract (Stage 2) of all identified studies were independently assessed for their relevance in answering the research question (details of the reviewers can be found in the Acknowledgments). The following short-term outcomes were assessed: incidence rates of severe RSV infection requiring medical treatment during the first or subsequent years of life, RSV hospitalization (RSVH) rates, length of stay (LOS) in hospital, RSVH-related outcomes [intensive care unit (ICU) admission, LOS in ICU, requirement for, and duration of, mechanical ventilation, non-invasive ventilation and oxygen], case-fatality rate, and risk factors for severe RSV infection requiring hospitalization. The following long-term outcomes were assessed: subsequent respiratory disease, including recurrent wheezing and asthma up to adulthood ( $\leq 18$ years) following severe RSV infection in infancy. Other outcomes assessed were the effectiveness of palivizumab in reducing RSVH rates and associated morbidity, long-term sequelae and mortality in different subgroups of children with or without CLD/BPD, and future developments in RSV research including genetic phenotypes and polymorphisms.

All studies marked in Phase 1 as potentially relevant were then independently assessed at the full-text level by the same two reviewers for inclusion (Phase 2). Any disagreements were resolved after discussion with a third reviewer (B. Rodgers-Gray) and X. Carbonell-Estrany. The search results were compiled into a spreadsheet, including documentation of the reason(s) for excluding a study. Data were extracted for all identified articles by one reviewer and quality-checked by the second reviewer, and a written report was produced (Supplementary Material 2-Data Extraction Table). The authors reviewed the search results and report, made 
any additions and amendments, and all approved the final list of studies for inclusion. For each systematic literature review, we fully documented the inclusion and exclusion processes using a PRISMA flowchart that detailed the number of included and excluded articles.

\section{Data Synthesis}

Included publications were graded according to the Oxford Centre for Evidence-Based Medicine Levels of Evidence [22, 23]: level 1 evidence (local and current random sample surveys [or censuses]); level 2 evidence (systematic review of surveys that allow matching to local circumstances); level 3 evidence (local non-random sample); level 4 evidence (case series). For randomized, controlled trials, a quality assessment for each citation was carried out using the five-point $(1=$ low quality; $5=$ high quality) Jadad Scale [24]. For each study, we also conducted a risk of bias assessment using the RTI Item Bank (score of $1=$ very high risk of bias; score of $12=$ very low risk of bias) for observational studies [25] and the Cochrane Collaboration's tool for assessing risk of bias for randomized clinical trials [26].

\section{METHODS USED FOR CURRENT STUDY: OVERALL EPIDEMIOLOGY AND BURDEN OF RSV}

The target population for the first systematic literature review in our series was previously healthy term children or studies with mixed populations of term and preterm $(<37 \mathrm{wGA})$ children and those with comorbidities. We excluded studies that focused exclusively on particular subgroups, such as preterm infants $(<37$ wGA), those with CLD/BPD or CHD, or other medical conditions (e.g. Down syndrome), since this systematic literature review considers the overall epidemiology and burden of RSV in infants and children. However, studies were included if a proportion of the study population had comorbidities.

In this first systematic literature review, we sought to answer the following question:

1. What is the overall epidemiology and disease burden of severe RSV infection in Western countries, and what are the associated risk factors for RSVH?

We performed a literature search using the following combination of search terms and limits: "RSV" OR "respiratory syncytial virus" AND "hospitalization" AND "epidemiology" OR "disease burden" OR "burden" OR "risk" OR "risk factor" AND "limits: human, infant aged up to 1 year; child (unspecified age)". "Bronchiolitis" and "pneumonia" were captured as part of the MeSH terms. It is recognized that while some relevant articles might have been missed by the searches, we are confident that the combined Boolean operators "AND" and "OR" of the key text words and index terms precisely captured the vast majority of relevant citations which were pertinent for this evidence-based review. The short-term outcomes of interest for this review included hospitalization rates due to severe RSV, hospital LOS, ICU admission and LOS, oxygen requirement, need for and duration of mechanical ventilation and/or non-invasive ventilation, case-fatality rates, and risk factors (including biological, environmental and social) for severe RSV infection requiring hospital admission.

\section{Statement of Ethics Compliance}

The analysis in this review article is based on previously published studies and does not 
involve any new studies of human subjects performed by any of the authors.

\section{RESULTS AND DISCUSSION}

\section{Articles Selected}

A total of 98 publications were included in the final review: 90 identified from the database searches and a further 8 from reference lists/ other sources (Fig. 1). Data extraction tables for all 98 studies, including evidence grades and risk of bias assessments, can be found in the online supplement (Supplementary Material 2-Data Extraction Table).

\section{Incidence of RSV Hospitalization}

RSV has been associated with $12-63 \%$ of all acute respiratory infections (ARIs) $[12,16,27-40]$ and $19-81 \%$ of viral ARIs causing hospitalization in infants and children [41-57] (Table 2). Importantly, while high-risk groups are particularly vulnerable to severe infection and are often the focus of study, the majority (typically $>70 \%$ ) of children

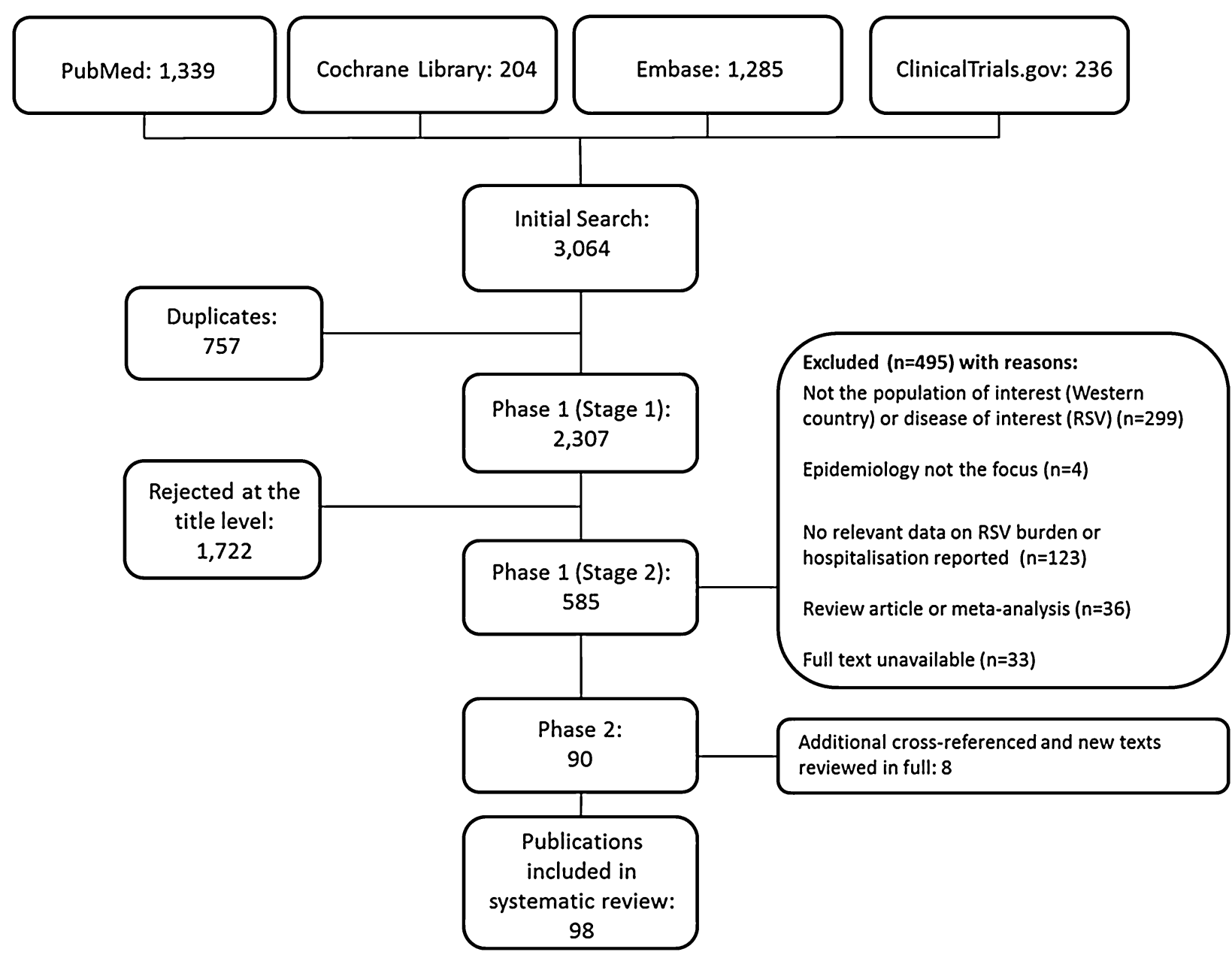

Fig. 1 PRISMA flow diagram: overall epidemiology and burden of severe respiratory syncytial virus (RSV) infection. The third reviewer (B. Rodgers-Gray) and X.
Carbonell-Estrany were not required to resolve any disagreements during the review process 
Table 2 Epidemiology of hospitalizations for RSV-associated bronchiolitis in infants and children

\begin{tabular}{|c|c|c|c|c|}
\hline Study & Country & Design & $\begin{array}{l}\text { \% RSV/total } \\
\text { ARIs }\end{array}$ & $\begin{array}{l}\text { Hospitalization } \\
\text { rate }\end{array}$ \\
\hline Resch (2002) [57] & Austria & $\begin{array}{l}\text { 1-year prospective study } \\
\text { (1999-2000); single center; all } \\
\text { children <2 years hospitalized with } \\
\text { viral U/LRTIs (included high-risk } \\
\text { children [preterm, CHD]) }\end{array}$ & $21 \%^{\mathrm{b}}$ & Term: 7/1000/year \\
\hline Resch (2000) [56] & Austria & $\begin{array}{l}\text { 4-year retrospective study } \\
\text { (1994-1998); single center; all } \\
\text { children hospitalized with viral } \\
\text { U/LRTIs (included high-risk } \\
\text { children [preterm, CHD, } \\
\text { pulmonary disease, neurological } \\
\text { disease]) }\end{array}$ & $26.7 \%^{\mathrm{b}}$ & Term: 6/1000/season \\
\hline $\begin{array}{l}\text { Santibanez (2012) } \\
\text { [36] }\end{array}$ & Canada & $\begin{array}{l}\text { 2-year retrospective study } \\
\text { (2008-2010); regional; all } \\
\text { hospitalized children }<19 \text { years old } \\
\text { with LRTI and RSV/LRTI } \\
\text { (included high-risk children and } \\
\text { infants [preterm, CHD, BPD/ } \\
\text { CLD]) }\end{array}$ & $29.5 \%$ & NR \\
\hline $\begin{array}{l}\text { Mlinaric-Galinovic } \\
\text { (2009) [46] }\end{array}$ & Croatia & $\begin{array}{l}\text { 11-year retrospective study } \\
\text { (1994-2005); national; all children } \\
<10 \text { years old hospitalized with an } \\
\text { ARI }\end{array}$ & $32.2 \%$ & NR \\
\hline $\begin{array}{l}\text { Haerskjold (2015) } \\
\text { [2] }\end{array}$ & Denmark & $\begin{array}{l}\text { 7-year population-based cohort study } \\
\text { (1997-2003); } 6 \text { national registries; } \\
\text { infants } \leq 24 \text { months old (included } \\
\text { high-risk infants [preterm, chronic } \\
\text { disease (e.g. congenital } \\
\text { malformations, intestinal lung } \\
\text { disease, chromosomal } \\
\text { abnormalities)]) }\end{array}$ & $\begin{array}{l}\text { Only RSV cases } \\
\text { enrolled }\end{array}$ & $\begin{array}{l}<2 \text { years: } 14.9 / 1000 \text { years } \\
\text { at risk }\end{array}$ \\
\hline $\begin{array}{l}\text { Kristensen (1998) } \\
\quad[83]\end{array}$ & Denmark & $\begin{array}{l}\text { 6-month population-based, } \\
\text { retrospective study (1995-1996); } \\
\text { regional; infants }<6 \text { months old } \\
\text { (included high-risk infants } \\
\text { [preterm, CHD, BPD/CLD, } \\
\text { neurologic disease, other]) }\end{array}$ & NR & $\begin{array}{l}<6 \text { months: } 34 / 1000 / \\
\text { season }\end{array}$ \\
\hline
\end{tabular}


Table 2 continued

\begin{tabular}{|c|c|c|c|c|}
\hline Study & Country & Design & $\begin{array}{l}\text { \% RSV/total } \\
\text { ARIs }\end{array}$ & $\begin{array}{l}\text { Hospitalization } \\
\text { rate }\end{array}$ \\
\hline $\begin{array}{l}\text { El-Hajje (2008) } \\
\quad[48]\end{array}$ & France & $\begin{array}{l}\text { 3-year prospective study } \\
\text { (2002-2004); single center; } \\
\text { children < } 16 \text { years old admitted } \\
\text { with an ARI, fever or asthma }\end{array}$ & $80.8 \%^{\mathrm{b}}$ & NR \\
\hline Weigl (2001) [29] & Germany & $\begin{array}{l}\text { 3-year retrospective study } \\
\text { (1996-1999); city; children } \\
\text { <16 years old admitted with an } \\
\text { ARI }\end{array}$ & $12.1 \%$ & $<2$ years: $7.25 / 1000$ \\
\hline Tsolia (2003) [84] & Greece & $\begin{array}{l}\text { 4-year retrospective/prospective } \\
\text { (1997-2000); regional; infants } \\
<12 \text { months old admitted with } \\
\text { bronchiolitis (included high-risk } \\
\text { infants [preterm infants } \leq 36 \mathrm{wGA} \text {, } \\
\text { CHD, and other underlying } \\
\text { illnesses]) }\end{array}$ & $61.5 \%$ & NR \\
\hline $\begin{array}{l}\text { Constantopoulos } \\
\text { (2002) [27] }\end{array}$ & Greece & $\begin{array}{l}\text { 2-year prospective, epidemiological } \\
\text { study (1999-2000); } 4 \text { regions; } \\
\text { children } \leq 2 \text { years old admitted } \\
\text { with ARI (included healthy } \\
\text { full-term infants and high-risk } \\
\text { infants [preterm, CHD, BPD/ } \\
\text { CLD]) }\end{array}$ & $33.1 \%$ & NR \\
\hline $\begin{array}{l}\text { Frassanito (2015) } \\
\quad[31]\end{array}$ & Italy & $\begin{array}{l}\text { 5-year retrospective study } \\
\text { (2009-2014); single center; } \\
\text { children <3 years old admitted } \\
\text { with a viral ARI }\end{array}$ & $54.2 \%$ & NR \\
\hline Ferrara (2014) [32] & Italy & $\begin{array}{l}\text { 9-year retrospective study } \\
\text { (2004-2013); single center; infants } \\
<12 \text { months old admitted with } \\
\text { bronchiolitis }\end{array}$ & $31.3 \%$ & NR \\
\hline $\begin{array}{l}\text { Zuccotti (2011) } \\
\qquad[30]\end{array}$ & Italy & $\begin{array}{l}\text { 12-month prospective, } \\
\text { epidemiological study } \\
\text { (2008-2009); 2-center; children } \\
\text { <15 years old admitted with an } \\
\text { ARI }\end{array}$ & $34.1 \%$ & NR \\
\hline
\end{tabular}


Table 2 continued

\begin{tabular}{|c|c|c|c|c|}
\hline Study & Country & Design & $\begin{array}{l}\% \mathrm{RSV} / \text { total } \\
\text { ARIs }\end{array}$ & $\begin{array}{l}\text { Hospitalization } \\
\text { rate }\end{array}$ \\
\hline $\begin{array}{l}\text { Corsello (2008) } \\
\text { [38] }\end{array}$ & Italy & $\begin{array}{l}\text { 7-month prospective, surveillance } \\
\text { study (2005-2006); regional; } \\
\text { children }<2 \text { years old admitted } \\
\text { with LRTI (included infants born } \\
<36 \text { wGA) }\end{array}$ & $40.9 \%$ & NR \\
\hline Medici (2006) [50] & Italy & $\begin{array}{l}4 \text { year prospective, observational } \\
\text { study (2000-2004); national; } \\
\text { children } \leq 4 \text { years old admitted for } \\
\text { an ARI (included previously } \\
\text { healthy term and at-risk }[\leq 35 \\
\text { wGA BPD/CLD] infants) }\end{array}$ & $49 \%$ & NR \\
\hline Lanari (2002) [33] & Italy & $\begin{array}{l}\text { 6-month prospective, epidemiological } \\
\text { study (1999-2000); multicenter; } \\
\text { children }<2 \text { years old admitted for } \\
\text { LRTI (included preterm infants } \\
\text { <36 wGA) }\end{array}$ & $40.6 \%$ & NR \\
\hline $\begin{array}{l}\text { Zomer-Kooijker } \\
\text { (2014) [51] }\end{array}$ & Netherlands & $\begin{array}{l}\text { 5-year prospective birth cohort study } \\
\text { (2003-2005 and 2006-2007); } \\
\text { single center; infants }<12 \text { months } \\
\text { old (all previously healthy term } \\
\text { infants) }\end{array}$ & $\begin{array}{l}\text { Only RSV cases } \\
\text { enrolled }\end{array}$ & $<1$ year: $8.4 / 1000 /$ year \\
\hline $\begin{array}{l}\text { Gooskens (2014) } \\
\text { [47] }\end{array}$ & Netherlands & $\begin{array}{l}\text { 2-year retrospective cohort study } \\
\text { (2006-2007); single center; } \\
\text { children <18 years old admitted } \\
\text { with an ARI (included preterm } \\
\text { infants and infants with bronchial } \\
\text { hyper-responsiveness and } \\
\text { cardiovascular disorders) }\end{array}$ & $31 \%^{\mathrm{b}}$ & NR \\
\hline Fjaerli (2004) [7] & Norway & $\begin{array}{l}\text { 8-year retrospective, population-based } \\
\text { study (1993-2000); single center/ } \\
\text { region; children <2 years old } \\
\text { admitted with bronchiolitis } \\
\text { (included at-risk infants [preterm, } \\
\text { Trisomy 21, CHD]) }\end{array}$ & $\begin{array}{l}\text { Only RSV cases } \\
\text { enrolled }\end{array}$ & $\begin{array}{l}<12 \text { months: } 21.7 / 1000 / \\
\text { year } \\
\text { 1-2 years: } 6.8 / 1000 / \text { year } \\
\text { <2 years: } 14.1 / 1000 / \text { year }\end{array}$ \\
\hline
\end{tabular}


Table 2 continued

\begin{tabular}{|c|c|c|c|c|}
\hline Study & Country & Design & $\begin{array}{l}\text { \% RSV/total } \\
\text { ARIs }\end{array}$ & $\begin{array}{l}\text { Hospitalization } \\
\text { rate }\end{array}$ \\
\hline Flores (2004) [85] & Portugal & $\begin{array}{l}\text { 2-year prospective study } \\
\text { (2000-2002); single center; } \\
\text { children }<3 \text { years old admitted } \\
\text { with acute bronchiolitis (included } \\
\text { high-risk children [preterm }<36 \\
\text { wGA, pulmonary disease]) }\end{array}$ & $60.9 \%$ & NR \\
\hline $\begin{array}{l}\text { Tatochenko (2010) } \\
\text { [52] }\end{array}$ & $\begin{array}{l}\text { Russian } \\
\text { Federation }\end{array}$ & $\begin{array}{l}\text { 6-month prospective, observational } \\
\text { study (2008-2009); multicenter; } \\
\text { children } \leq 2 \text { years old admitted } \\
\text { with LRTI (included high-risk } \\
\text { children [preterm, CLD/BPD, } \\
\text { CHD]) }\end{array}$ & $37.9 \%$ & NR \\
\hline $\begin{array}{l}\text { Gil-Prieto (2015) } \\
\quad[4]\end{array}$ & Spain & $\begin{array}{l}\text { 15-year observational, retrospective } \\
\text { survey (1997-2011); included } \\
\text { >98\% Spanish hospitals; children } \\
\text { <5 years old admitted with } \\
\text { bronchiolitis (included high-risk } \\
\text { children [preterm, congenital } \\
\text { cardiopathies/defects, BPD]) }\end{array}$ & $\begin{array}{l}\text { Only RSV cases } \\
\text { enrolled }\end{array}$ & $\begin{array}{l}<2 \text { years: } 24.1 / 1000 / \text { year } \\
<5 \text { years: } 10.7 / 1000 / \text { year }\end{array}$ \\
\hline Hervás (2012) [12] & Spain & $\begin{array}{l}\text { 12-year retrospective study } \\
\text { (1995-2006); single center/region; } \\
\text { children } \leq 2 \text { years old admitted } \\
\text { with acute bronchiolitis (included } \\
\text { high-risk children [preterm, CHD, } \\
\text { atelectasis/condensation, urinary } \\
\text { tract infection]) }\end{array}$ & $62.7 \%$ & $\leq 2$ years: $55 / 1000^{\mathrm{a}}$ \\
\hline $\begin{array}{r}\text { García-García } \\
\text { (2012) [42] }\end{array}$ & Spain & $\begin{array}{l}\text { 6-year prospective study } \\
\text { (2004-2010); single center; } \\
\text { children <14 years old admitted } \\
\text { with community-acquired } \\
\text { pneumonia }\end{array}$ & $41.6 \%^{b}$ & NR \\
\hline $\begin{array}{l}\text { Salvador García } \\
\text { (2012) [28] }\end{array}$ & Spain & $\begin{array}{l}\text { 1-year prospective study } \\
\text { (2008-2009); region; children } \\
\text { <18 months old admitted with } \\
\text { bronchiolitis }\end{array}$ & $56.4 \%$ & NR \\
\hline Calvo (2010) [39] & Spain & $\begin{array}{l}\text { 3-year prospective study } \\
\text { (2005-2008); single center; } \\
\text { children }<2 \text { years old admitted } \\
\text { with acute viral bronchiolitis }\end{array}$ & $61.3 \%$ & NR \\
\hline
\end{tabular}


Table 2 continued

\begin{tabular}{|c|c|c|c|c|}
\hline Study & Country & Design & $\begin{array}{l}\text { \% RSV/total } \\
\text { ARIs }\end{array}$ & $\begin{array}{l}\text { Hospitalization } \\
\text { rate }\end{array}$ \\
\hline $\begin{array}{l}\text { Artiles-Campelo } \\
\text { (2006) [43] }\end{array}$ & Spain & $\begin{array}{l}\text { 3-year prospective study } \\
\text { (2002-2005); single center; } \\
\text { children < } 14 \text { years old admitted } \\
\text { with an ARI }\end{array}$ & $74.5 \%^{\mathrm{b}}$ & NR \\
\hline $\begin{array}{l}\text { Díez Domingo } \\
\quad(2006)[40]\end{array}$ & Spain & $\begin{array}{l}\text { 2-year retrospective study } \\
\text { (2001-2002); province; children } \\
\text { <2 years old admitted with } \\
\text { bronchiolitis }\end{array}$ & $42.2 \%$ & $\begin{array}{l}<12 \text { months: } 40.2 / 1000 / \\
\text { year }^{\mathrm{c}}\end{array}$ \\
\hline Vicente (2003) [6] & Spain & $\begin{array}{l}\text { 4-year retrospective, population-based } \\
\text { study (1996-2000); single center/ } \\
\text { province; children }<5 \text { years old } \\
\text { admitted with an ARI }\end{array}$ & $59.1 \%$ & $\begin{array}{l}<6 \text { months: } 37 / 1000 / \text { year } \\
<12 \text { months: } 25 / 1000 / \\
\text { year }\end{array}$ \\
\hline $\begin{array}{l}\text { Svensson }(2015) \\
\quad[61]\end{array}$ & Sweden & $\begin{array}{l}\text { 7-year retrospective study } \\
\text { (2004-2011); regional; children } \\
<5 \text { years old with an RSV infection } \\
\text { (included high-risk children } \\
\text { [wheezy bronchitis, CHD, Down } \\
\text { syndrome, small for gestational } \\
\text { age]) }\end{array}$ & $\begin{array}{l}\text { Only RSV cases } \\
\text { enrolled }\end{array}$ & $\begin{array}{l}<12 \text { months: } 17.4 / 1000 / \\
\text { year } \\
\text { 1-4 years: } 0.6 / 1000 / \text { year } \\
\text { <5 years: } 4.2 / 1000 / \text { year }\end{array}$ \\
\hline $\begin{array}{l}\text { Erikksson (2002) } \\
\text { [86] }\end{array}$ & Sweden & $\begin{array}{l}\text { 12-year retrospective study } \\
\text { (1987-1998); single center; } \\
\text { children admitted with confirmed } \\
\text { RSV infection (included high-risk } \\
\text { children [preterm, BPD/CLD, } \\
\text { cardiac malformation, respiratory } \\
\text { malformation, other chronic } \\
\text { disease]) }\end{array}$ & $\begin{array}{l}\text { Only RSV cases } \\
\text { enrolled }\end{array}$ & $0.8-0.14 / 100 /$ year \\
\hline Bicer (2013) [52] & Turkey & $\begin{array}{l}\text { 1-year retrospective cohort study } \\
\text { (2010-2011); single center; } \\
\text { children <9 years old admitted } \\
\text { with an ARI }\end{array}$ & $32 \%^{b}$ & NR \\
\hline $\begin{array}{l}\text { Hacımustafaoğlu } \\
\text { (2013) [54] }\end{array}$ & Turkey & $\begin{array}{l}\text { 1-year study; multicenter; children } \\
\leq 2 \text { years old admitted with LRTI }\end{array}$ & $37.9 \%$ & 7.8/1000/year \\
\hline
\end{tabular}


Table 2 continued

\begin{tabular}{|c|c|c|c|c|}
\hline Study & Country & Design & $\begin{array}{l}\text { \% RSV/total } \\
\text { ARIs }\end{array}$ & $\begin{array}{l}\text { Hospitalization } \\
\text { rate }\end{array}$ \\
\hline $\begin{array}{l}\text { Turkish Neonatal } \\
\text { Society (2012) } \\
\text { [55] }\end{array}$ & Turkey & $\begin{array}{l}\text { 2-year prospective, epidemiological } \\
\text { study }(2008-2010) \text {; multicenter; } \\
\text { children }<2 \text { years old with } \\
\text { diagnosis of respiratory failure due } \\
\text { to LRTI (excluded children with } \\
\text { immunodeficiency, CF or } \\
\text { congenital or acquired disorders } \\
\text { affecting respiratory system) }\end{array}$ & $16.9 \%$ & NR \\
\hline $\begin{array}{l}\text { Ajayi-Obe (2008) } \\
\text { [37] }\end{array}$ & UK & $\begin{array}{l}\text { 2-year prospective, descriptive study } \\
\text { (2002-2003 and 2003-2004); } \\
\text { single center; children <6 years old } \\
\text { presenting with influenza-like } \\
\text { illness (included high-risk children } \\
\text { [asthma, CHD, BPD, CF, sickle } \\
\text { cell disease, chronic renal disease, } \\
\text { thalassemia immunodeficiencies]) }\end{array}$ & $\begin{array}{l}19.1 \% \\
\quad(2002-2003)^{b} \\
27 \% \\
\quad(2003-2004)^{b}\end{array}$ & $\begin{array}{l}<6 \text { months: } 56.2 / 1000 \\
\text { person-years } \\
<12 \text { months: } 42.7 / 1000 \\
\text { person-years } \\
2-3 \text { years: } 7.28 / 1000 \\
\text { person-years } \\
4-5 \text { years: } 0.67 / 1000 \\
\text { person-years } \\
<6 \text { years: } 12.87 / 1000 \\
\text { person-years }\end{array}$ \\
\hline $\begin{array}{l}\text { Deshpande (2003) } \\
\quad[63]\end{array}$ & UK & $\begin{array}{l}\text { 3-year retrospective, population-based } \\
\text { study (1996-1999); county; } \\
\text { children <2 years old admitted } \\
\text { with a respiratory illness (included } \\
\text { high-risk children [preterm, BPD/ } \\
\text { CLD]) }\end{array}$ & $58.8 \%$ & $<2$ years: $16.3 / 1000^{\mathrm{a}}$ \\
\hline $\begin{array}{l}\text { Müller-Pebody } \\
\text { (2002) [34] }\end{array}$ & UK & $\begin{array}{l}\text { 3-year retrospective study } \\
\text { (1995-1998); national; children } \\
\text { <5 years old admitted with LRTI } \\
\text { (included high-risk children } \\
\text { [preterm, BPD/CLD]) }\end{array}$ & $17.5 \%$ & $\begin{array}{l}<12 \text { months: } 28.3 / 1000 / \\
\text { year } \\
1-4 \text { years: } 1.3 / 1000 / \text { year }\end{array}$ \\
\hline Hall (2013) [16] & US & $\begin{array}{l}\text { 5-year prospective, population-based } \\
\text { study (2000-2005); } 3 \text { counties; } \\
\text { children }<2 \text { years old admitted } \\
\text { with an ARI (included preterm } \\
\text { infants }<37 \text { wGA and infants with } \\
\text { comorbid conditions) }\end{array}$ & $26 \%$ & $\begin{array}{l}1 \text { month: } 25.9 / 1000^{\mathrm{a}} \\
3 \text { months: } 10.3 / 1000^{\mathrm{a}} \\
6 \text { months: } 4.1 / 1000^{\mathrm{a}} \\
12 \text { months: } 3.2 / 1000^{\mathrm{a}} \\
18 \text { months: } 2.6 / 1000^{\mathrm{a}} \\
<2 \text { years: } 5.2 / 1000^{\mathrm{a}}\end{array}$ \\
\hline
\end{tabular}


Table 2 continued

\begin{tabular}{|c|c|c|c|c|}
\hline Study & Country & Design & $\begin{array}{l}\% \mathrm{RSV} / \text { total } \\
\text { ARIs }\end{array}$ & $\begin{array}{l}\text { Hospitalization } \\
\text { rate }\end{array}$ \\
\hline $\begin{array}{l}\text { Hasegawa (2013) } \\
\quad[70]\end{array}$ & US & $\begin{array}{l}\text { 4-year serial, cross-sectional analysis of } \\
\text { a nationally representative sample } \\
\text { of children (2000, 2003, 2006, } \\
\text { 2009); national; children }<2 \text { years } \\
\text { old hospitalized with bronchiolitis } \\
\text { (included high-risk children } \\
\text { [preterm or } \geq 1 \text { complex medical } \\
\text { condition]) }\end{array}$ & NR & $\begin{array}{l}<2 \text { years: } 17.9 / 1000 \\
\text { person-years }^{c}(2000) ; \\
14.8 / 1000 \text { person-years } \\
(2009)\end{array}$ \\
\hline $\begin{array}{l}\text { Stockman (2012) } \\
\text { [35] }\end{array}$ & US & $\begin{array}{l}\text { 10-year retrospective study } \\
\text { (1997-2006); national; children } \\
<5 \text { years old admitted for LRTI } \\
\text { (included high-risk children } \\
\text { [preterm, CHD, chronic } \\
\text { respiratory distress]) }\end{array}$ & $\sim 24 \%$ & $\begin{array}{l}0-2 \text { months: } 48.9 / 1000 / \\
\text { year } \\
\text { 3-5 months: } 28.4 / 1000 / \\
\text { year } \\
<12 \text { months: } 13.4 / 1000 / \\
\text { year } \\
1-2 \text { years: } 5.0 / 1000 / \text { year } \\
<5 \text { years: } 6 / 7 / 1000 / \text { year }\end{array}$ \\
\hline Zhou (2012) [49] & US & $\begin{array}{l}\text { 16-year retrospective surveillance } \\
\text { study (1993-2008); } 13 \text { states; all } \\
\text { ages including adults admitted with } \\
\text { influenza or RSV }\end{array}$ & $19.5 \%^{b}$ & $\begin{array}{l}<12 \text { months: } 23.5 / 1000 \\
\text { person-years } \\
1-4 \text { years: } 1.78 / 1000 \\
\text { person-years }\end{array}$ \\
\hline García (2010) [44] & US & $\begin{array}{l}\text { 6-year retrospective study } \\
\text { (2002-2007); single center/region; } \\
\text { children <2 years old admitted } \\
\text { with bronchiolitis (included } \\
\text { high-risk children [prematurity, } \\
\text { CHD, BPD/CLD, Trisomy 21, } \\
\text { congenital syndromes, } \\
\text { immunodeficiencies, CF, } \\
\text { neuromuscular disorders, } \\
\text { respiratory tract disorders]) }\end{array}$ & $66 \% \%^{b}$ & NR \\
\hline Iwane (2004) [45] & US & $\begin{array}{l}\text { 12-month prospective, active, } \\
\text { population-based, surveillance study } \\
\text { (2000-2001); } 2 \text { counties; children } \\
<5 \text { years old admitted with an ARI } \\
\text { (included children with chronic } \\
\text { conditions) }\end{array}$ & $20 \%^{\mathrm{b}}$ & $<5$ years: $3.5 / 1000 /$ year \\
\hline
\end{tabular}


Table 2 continued

\begin{tabular}{|c|c|c|c|c|}
\hline Study & Country & Design & $\begin{array}{l}\text { \% RSV/total } \\
\text { ARIs }\end{array}$ & $\begin{array}{l}\text { Hospitalization } \\
\text { rate }\end{array}$ \\
\hline Leader (2003) [62] & US & $\begin{array}{l}\text { 3-year retrospective study } \\
\text { (1997-2000); national; infants } \\
\leq 12 \text { months old admitted with } \\
\text { RSV (included high-risk children } \\
\text { [premature, CHD, BPD/CLD, } \\
\text { chromosomal abnormalities]) }\end{array}$ & $\begin{array}{l}\text { Only RSV cases } \\
\text { enrolled }\end{array}$ & $\leq 12$ months: $22.7 / 1000^{\mathrm{a}}$ \\
\hline
\end{tabular}

$A R I$ acute respiratory infection, $B P D$ bronchopulmonary dysplasia, $C F$ cystic fibrosis, $C H D$ congenital heart disease, $C L D$ chronic lung disease, $G A$ gestational age, $L R T I$ lower respiratory tract infection, $N R$ not reported, $R S V$ respiratory syncytial virus, $U / L R T I$ upper or lower respiratory tract infection, $w G A$ weeks' gestational age

a Overall hospitalization rate during study period

b Virally confirmed ARIs tested

c Bronchiolitis-related hospitalizations

hospitalized with RSV-related ARI/bronchiolitis had no underlying medical conditions $[14,16,44,56-58]$. To put the burden of RSV into context, in comparison to influenza, retrospective analyses show that RSV causes up to 16 times more hospitalizations and emergency department visits in children aged $<5$ years [49, 59, 60].

Hospitalization rates for RSV ARIs increase with decreasing age, peaking in the first few months of life $[4,6,7,16,34,35,37,56,61]$. Most retrospective studies have consistently shown RSVH to be the highest in the first year of life $[7,49,56,61]$. In the prospective study by Hall et al. [16], 1-month-old infants, consistently, were the most likely to be hospitalized, almost twice as often as the next two most at-risk groups: infants $<1$ month old and infants 2 months old. Incidence rates for confirmed RSVH in the first year of life in healthy term infants may be relatively low at $<1 \%$ [51], though around $2 \%$ of all infants including those with comorbidities are included $[61,62]$. More studies in healthy term infants are required. Overall, studies have found that between $75 \%$ and $90 \%$ of infants hospitalized with RSV were aged $\leq 12$ months, and between $44 \%$ and $83 \%$ were aged $\leq 6$ months $[6,7,12,16,44,56,63-65]$. Only a minority $(\leq 5 \%)$ of neonatally hospitalized children are rehospitalized for RSV infection [57, 66, 67].

Annual hospitalization rates for RSV-associated ARIs in the first year of life range from 3.2/1000/year [16] to 42.7/1000/ year [37], and decreased with increasing age to $0.6 / 1000 /$ year [61] to $1.78 / 1000 /$ year [49] in children aged 1-4 years (Table 2 ). The reported rates of RSVH, however, vary considerably between studies and across seasons within the same study.

\section{Incidence of RSV in Emergency Departments and Outpatient Settings}

There are very limited data available on the incidence and burden of RSV infection managed on an outpatient basis or in the emergency department $[14,60]$. Available studies suggest that the outpatient burden of RSV on healthcare resources has not been fully recognized by healthcare providers [14]. For example, a US study reported that only $3 \%$ of 
outpatients with RSV infection received the diagnosis of RSV-associated illness, as compared with $45 \%$ of inpatients $(P<0.001)$ [14]. Based on study data, RSV infection was estimated to result in one of 334 hospitalizations, one of 38 visits to an emergency department, and one of 13 visits to a primary care office each year in the US [14]. Another US study estimated that, at a national level, more children required an emergency department visit for a RSV infection compared with an influenza infection (21.5 vs. 10.2 visits per 1000 children/year, respectively) [60]. The total estimated number of workdays missed annually by caregivers of children who required emergency department care was 716,404 days for RSV infections [60].

\section{Trends in RSV Hospitalization Rates}

A number of studies have examined the national trends in RSVH rates among infants and children over the past two decades. While some studies conducted in the United States $[44,68]$ and Canada [69] throughout the 1980s and 1990s and the early 2000s have shown a rise in RSVH rates, other studies, both in the United States [70] and in France [71], conducted between 1998 and 2009, report a decrease in the incidence rate of bronchiolitis and RSVH. Further studies in the United States and Europe report that RSVH rates have remained relatively stable over similar time periods [7, 35, 72]. A firm conclusion on long-term time trends is not possible.

Season-on-season RSVH rates have been shown to vary by up to a factor of two to three in the same geographic region $[6,7,16]$. The length and start date of the RSV season can also vary from year to year [50, 73-75]. In temperate, northern hemisphere countries the season typically starts in October or November, peaks in December or February, and finishes in March or April [4, 6, 7, 16, 44, 50, 63]. Changes in surveillance programs, testing rates, the RSV tests used, and admission criteria within and between countries, as well as variations in disease severity and levels of circulating virus, all contribute to the varying incidences reported over time and highlight the need for rigorous, uniform, and ongoing data collection.

\section{Co-infections}

RSV has a high rate of co-infection with other respiratory viruses with a similar seasonal pattern, such as influenza, rhinovirus (RV), human metapneumovirus (hMPV) and human bocavirus (HBoV) [28, 41, 76, 77], and also with bacterial pathogens [78, 79]. Co-infections, most commonly dual infections, have been reported to occur in up to $50 \%$ of RSV-hospitalized children, [76, 78, 79]. In a prospective study of 2525 children aged $<14$ years by Calvo et al. [76], 599 RSV single infections and 326 RSV multiple infections were detected. The most frequent dual infection was between RSV and RV (120 episodes) and the second between RSV and HBoV (60 episodes). Other dual RSV infections included 3 episodes of RSV and hMPV and 11 episodes of RSV and influenza [76].

Co-infections represent a major confounder in burden of disease analyses of RSV infection $[78,79]$. At present, the clinical data available on co-infection, in terms of both the number of viruses involved and the severity of the condition, are variable and even, at times, contradictory [80]. Some studies have reported that multiple infections were associated with more severe disease and longer hospital stays than with single RSV infections [42, 76, 81]. Calvo et al. [76] found that hospitalization was longer for both RSV simple and RSV and HBoV 
co-infection, lasting about 1 day $(4.7$ vs. 3.8 days; $P<0.001$ ) longer than in simple $\mathrm{HBoV}$ infections. In contrast, Martinez-Roig et al. [80] found an inverse relationship between the number of viruses detected in nasopharyngeal aspirate, the need for oxygen therapy and hospitalization days. In this prospective study of 463 pediatric patients aged between 7 days and 15 years hospitalized with respiratory infections (none with risk factors for respiratory diseases), the most common co-infections were RV and RSV-B (10 cases) and RSV-A and RSV-B (5 cases). Hospitalization decreased with greater number of viruses $(P<0.001)$. Oxygen therapy was required by $26.75 \%$ ( 1 virus was detected in $55.34 \%$ of cases). A larger number of viruses resulted in less need for oxygen $(P<0.001)$. Ten cases required mechanical ventilation, 4 patients with bacterial co-infection and 5 with viral co-infection $(P=0.69)$. The most common co-infections were RV and RSV-B (10 cases) and RSV-A and RSV-B (5 cases) [80]. Findings from an earlier Canadian study of 742 children suspected to have a respiratory illness (33\% with a comorbidity) support equivalent disease severity between single virus infection and virus co-infection [82]. In multivariable analysis, virus co-infection did not affect admission to hospital after adjustment for age and the presence of underlying conditions [82].

\section{Morbidity and Mortality Associated with Severe RSV Infection}

\section{Morbidity}

Severe RSV infection in infants and children is associated with substantial morbidity and constitutes a considerable burden on healthcare systems [83]. Kristensen et al. [83] showed that, compared with infants without predisposing conditions (aged $<6$ months), infants with predisposing conditions were hospitalized longer (median 5 vs. 9 days; $P<0.001)$ and were treated longer with continuous positive airway pressure (CPAP) (median 4 vs. 7 days; $P<0.001$ ) and supplemental oxygen (median 3 vs. 5 days; $P=0.05)$. In term infants without predisposing conditions, age was a predictor of disease severity. Age correlated inversely with duration of oxygen therapy $(P<0.02)$, and infants treated with oxygen or CPAP were significantly younger than infants who did not receive respiratory support (oxygen: median age, 52 vs. 91 days; $P<0.001$; CPAP: median age, 51 vs. 90 days; $P=0.001$ ) [83]. Müller-Pebody et al. [34] observed that hospital LOS was significantly higher $(P<0.001)$ in high-risk children aged $<1$ year and in those aged 1-4 years. While Gijtenbeek et al. [88] observed higher hospitalization rates for confirmed RSV infection among preterm infants (32-36 wGA) than full-term infants, disease severity, length of hospitalization and the use of mechanical ventilation and oxygen were similar between gestational groups (Table 3).

Studies have shown that RSV ARI is associated with more severe disease than non-RSV ARI, resulting in a significant impact on healthcare resource utilization [12, 44, 57]. On average, infants spend 2-11 days in hospital for RSV ARIs, with around $2-12 \%$ being admitted to ICU (Table 3) $[4,6,7,12,27,29,34,39,41,44$, $56,57,61-64,70,84,87,88$ ]. Vicente et al. [6] reported that infants younger than 6 months had the longest duration of hospital stay. Perhaps unsurprisingly, different geographic regions have varying criteria for admission to hospital and subsequent management [89, 90], which can make comparisons of disease severity difficult. 


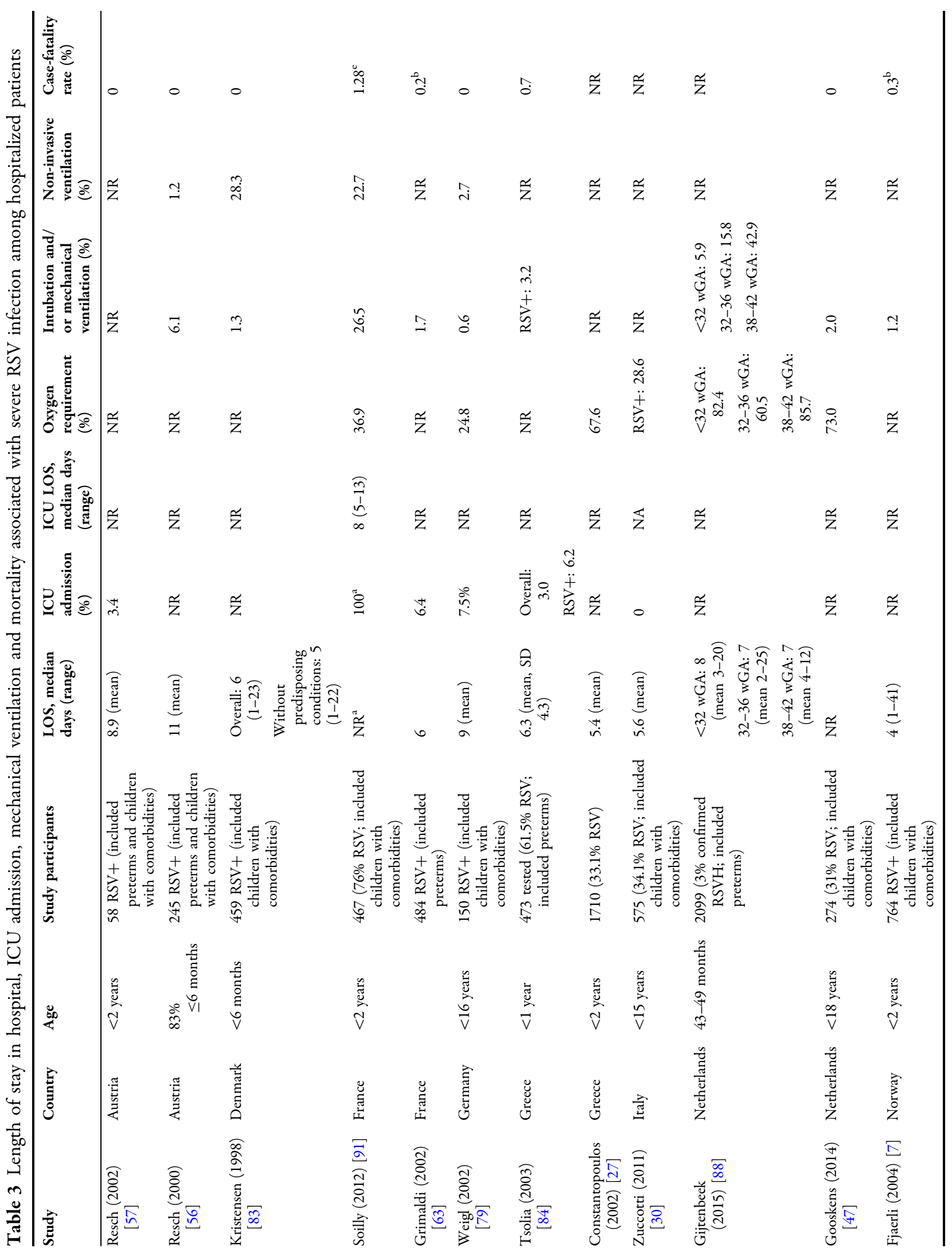




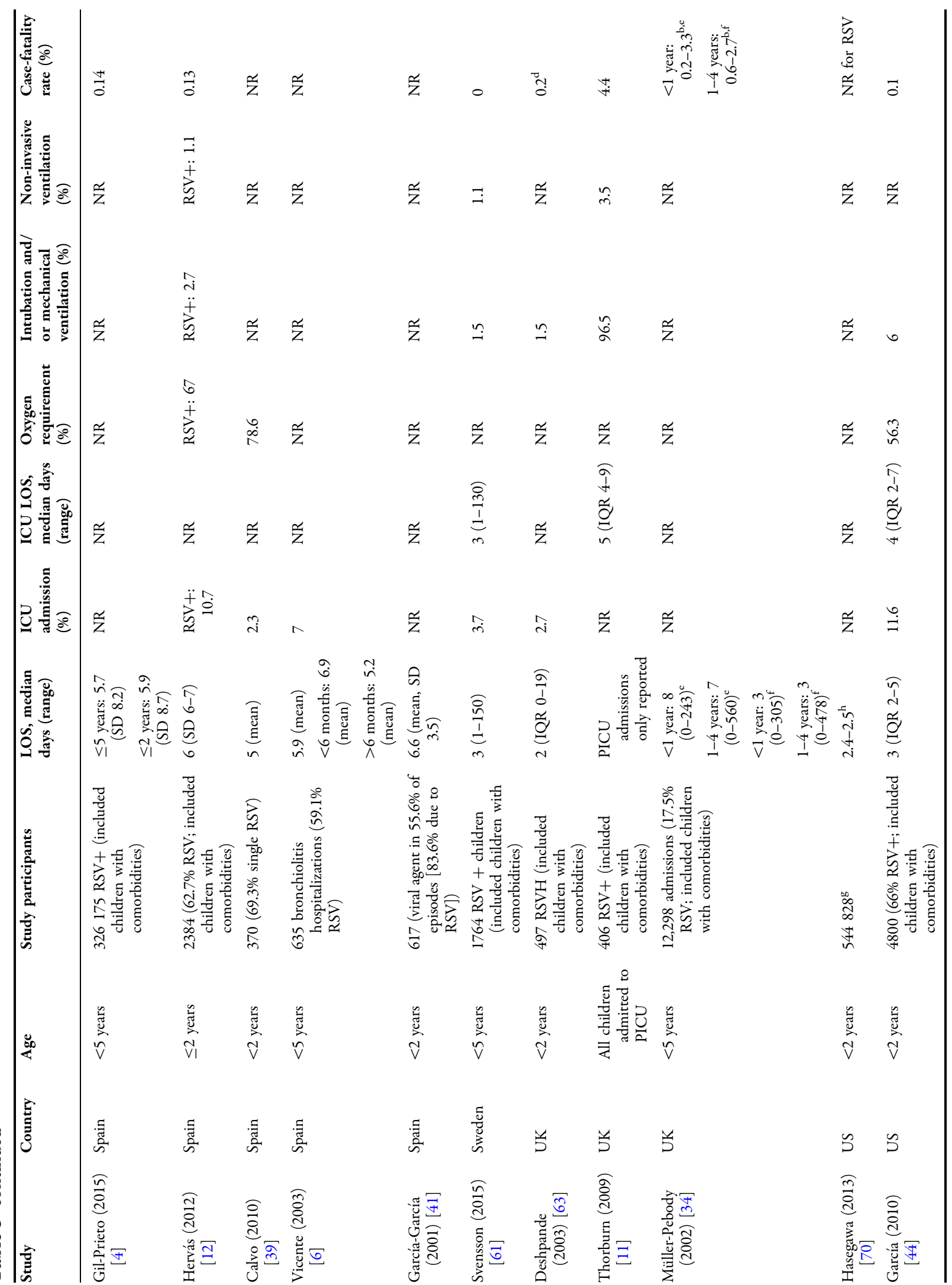




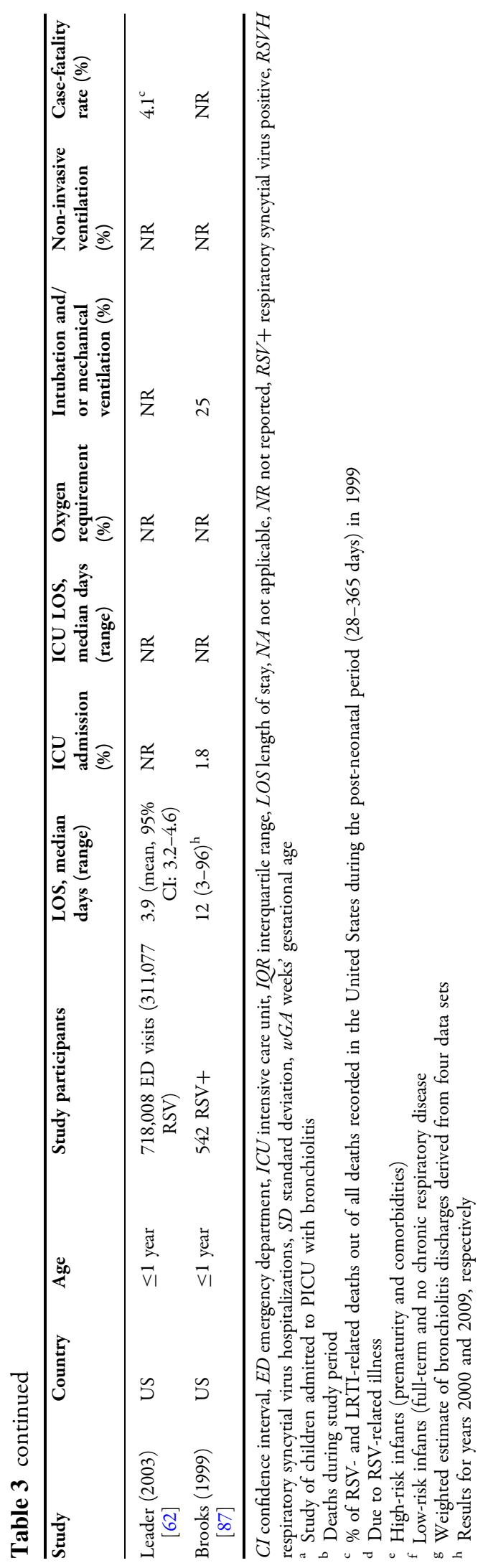

\section{Nosocomial RSV Infection}

There are limited published data on the epidemiology of nosocomial RSV infection, but in infants with comorbidities, it often follows a severe course of disease, sometimes resulting in mortality [11]. Typically, nosocomial RSV infection occurs at a low rate $(0.4-6 \%)$ [83, 92, 93]. A recent study conducted in 44 neonatal ICUs in Turkey reported a higher rate of nosocomial infection in newborns (9.6\%) [94]. Nosocomial RSV infection was shown to be more severe than community-acquired RSV in a prospective, multicenter, surveillance study undertaken in Germany over 6 consecutive seasons (1999-2005) [93]. The median LOS was 72 days [interquartile range (IQR) 31-131] for nosocomial RSV infection compared with 7 days (IQR 4-10) for community-acquired RSV $(P<0.001)$. Significantly more children were admitted to ICU $(52 \%$ vs. $9 \%$, respectively; $\quad P<0.001)$ and required mechanical ventilation $(56.7 \%$ vs. $12.6 \%$; $P<0.001)$ [93].

\section{Case-Fatality Rates}

The Global Burden of Disease study estimated that RSV accounted for $1.6 \%$ of deaths worldwide in 2010 [95]. Available data from the published literature suggest that the mortality rate for RSV is low in the West (typically $<0.5 \%$ ) $[4,7,12,44,56,57,63-65,96]$, whereas higher rates have been reported in low- and middle-income countries [97, 98]. Despite advances in neonatal care, rates of mortality have been shown to remain relatively stable over time. A study in the United States reported a case-fatality rate for bronchiolitis-associated deaths of infants aged $<1$ year of 2.2/100,000 live births in 1979 vs. 2.4/100,000 in 1997 [99]. In a recent 15 -year study in Spain, the case-fatality rate was $0.14 \%$, ranging from $0.11 \%$ in infants 
Table 4 Reported risk factors for severe RSV disease requiring hospitalization in otherwise healthy term and mixed populations of children

Independent risk factors for severe RSV infection requiring hospitalization

Presence of older sibling [2, 108]

Birth in proximity to RSV season $[16,109,110]$

Low birth weight $[108,110]$

Birth order [33, 109]

Male sex [108]

Young age ( $<6$ months) $[14,16,27,100]$

Exposure to smoking [86, 101, 108]

Maternal age [110]

Suburban residence [110]

Other risk factors associated with RSV hospitalization

Vitamin D deficiency [111]

Family history of atopy/atopic diagnosis [101, 112, 113]

Climatic factors and air pollution [114-116]

High altitude above $2500 \mathrm{~m}$ [104]

Socioeconomic status/parental education [117]

Delivery by cesarean section [105]

Level of evidence

Key statements/findings

In western countries, RSV has been associated with $12-63 \%$ of all ARIs and $19-81 \%$ of viral ARIs causing hospitalization in children

Annual hospitalization rates for RSV-associated ARIs ranged from 3.2/1000 children to 42.7/1000 in the first year of life, and decreased with decreasing age to $0.6 / 1000-1.78 / 1000$ in children $1-4$ years

Longitudinal studies have reported varying annual incidences of RSVH over time: some have reported an increase, some a decrease, and others relative stability

RSV co-infections are frequent (bacterial and viral co-infections have been reported in up to $50 \%$ of patients hospitalized with severe RSV ARI). However, the relationship with severity of disease is not clear

Infants spend an average of $2-11$ days in hospital for RSV ARIs, around $2-12 \%$ are admitted to the ICU, and the mortality rate is $<0.5 \%$ and limited to children with severe comorbidity

A number of risk factors have been independently associated with an increased risk for severe RSV disease in children, including: male sex; age $<6$ months; birth during the first half of the RSV season; crowding/siblings; and day-care exposure

Key areas for research

The current evidence base likely underestimates the true epidemiology and burden of severe RSV infection, particularly, the burden related to outpatient and emergency department visits requires further study

The impact of co-infections on the severity of RSV infections requires further definition and study

Mortality rates are mainly based on excess mortality estimates during the RSV season in a few studies. Refining global mortality estimates and defining associated risk factors require further study

Geographical differences in RSV epidemiology need to be defined

$A R I$ acute respiratory infection, $I C U$ intensive care unit, $R S V H$ respiratory syncytial virus hospitalization

${ }^{a}$ Level 1: local and current random sample surveys (or censuses); Level 2: systematic review of surveys that allow matching to local circumstances; Level 3: local non-random sample; Level 4: case series [22] 
aged 1 year to $0.18 \%$ in children aged 4 years [4]. In several studies, the case-fatality rate was higher among high-risk children, including those with BPD/CLD, CHD and immunodeficiency $[4,7,11,34]$.

\section{Risk Factors for RSV Hospitalization}

Several important independent risk factors for RSV hospitalization have been identified, including male gender, birth in proximity to RSV season, history of prematurity and tobacco smoke exposure (Table 4). Age, in particular, has been found to be a significant predictor in the severity of infection and RSV hospitalization, with the youngest age groups ( $<6$ months) more frequently hospitalized for a RSV infection $[14,16,27,100,101]$. In contrast, some risk factors, such as breastfeeding, have been found to be protective [33, 102]. While the predominance of severe RSV bronchiolitis in boys compared to girls is well known, its mechanism is not yet understood [103]. A study found that the interleukin-9 genetic polymorphism (rs2069885) has an opposite effect on the risk of severe RSV bronchiolitis in boys and girls [103]. Some lesser known risk factors reported in the literature include high altitude above $2500 \mathrm{~m}$ [104], maternal atopy [101] and delivery by cesarean section [105]. Black and aboriginal race has also been shown in some studies to be a risk factor for severe disease $[14,65,90,101,106]$, but not in others $[16,35,107]$. The reasons for these racial disparities, however, remain unclear, but may relate to social factors. Risk factors for RSV-related mortality have not been well described. Insights into risk factors that predispose infants to severe RSV infection and subsequent hospitalization are important to identify strategies to prevent infection, as well as to inform guidelines for palivizumab prophylaxis.

\section{Limitations}

There are a few key limitations when considering this review. With a remit to cover 20 years of study and research into the epidemiology and burden of RSV, it is recognized that improvements and changes in medical practice over time would influence the findings and conclusions drawn. The availability, type, and level of diagnostic testing for RSV undertaken over the review period is particularly relevant. Older studies-if any-predominantly used rapid antigen testing, shell vial culture, and viral culture, which are generally less sensitive than current PCR (polymerase chain reaction)-based assays. As described above, some studies, particularly the older ones, tended to describe the epidemiology and burden of bronchiolitis or ARIs without a totally accurate measure of how much of that burden or any additional burden related to RSV. We consider that there is a really important economical impact of RSV infection on emergency departments and outpatients, on co-infections, and on RSV-specific mortality rates. Unfortunately, there are also limited economical data of outpatient RSV economical burden For this reason, this review may underestimate the true burden of RSV.

\section{CONCLUSIONS}

Despite the availability of current preventative measures, the findings of this review confirm that RSVH still represents a large burden of disease in Western countries since it is associated with significant morbidity. Further studies focused on the epidemiology and the incidences of RSV ARIs to determine the true 
burden of disease, particularly in the outpatient setting, are needed. Well-coordinated surveillance programs within and across Western countries involving centers and hospitals with robust electronic medical records systems for patient identification and that test a high proportion of hospitalized children or visiting outpatients with respiratory illness for RSV, would be the ideal. Also, the development of more effective preventive strategies in high-risk children, as well as in healthy populations, is essential to reduce the overall burden of RSV ARI, which will be the focus of a subsequent paper in the REGAL series.

\section{ACKNOWLEDGMENTS}

Dr Joanne Smith, Julie Blake (Reviewers 1 and 2) and Dr Barry Rodgers-Gray (Reviewer 3), from Strategen Limited, undertook the systematic review following the protocol approved by the authors. AbbVie provided funding to Strategen to undertake the systematic review. All named authors meet the International Committee of Medical Journal Editors (ICMJE) criteria for authorship for this manuscript, take responsibility for the integrity of the work as a whole, and have given final approval to the version to be published. Editorial assistance in the preparation of this manuscript was provided by Julie Blake and Dr Barry Rodgers-Gray. J. Blake and B. Rodgers-Gray developed a first draft of the manuscript, based on the results of the systematic review and input/approval from all authors, which was initially edited by $\mathrm{X}$. Carbonell-Estrany and L. Bont and then circulated among the other authors for input, further edits and subsequent approval. Support for this assistance was funded by AbbVie. AbbVie had the opportunity to review and comment on the completed manuscript but final editorial control rested fully with the authors. Article processing charges for this study were funded by Abbvie.

Disclosures. The institute of L. Bont received money for investigator initiated studies by MeMed, Astra Zeneca, AbbVie, and Janssen. The institute of $\mathrm{L}$. Bont received money for consultancy by Astra Zeneca, AbbVie, MedImmune, Janssen, Gilead and Novavax. P. Checchia has acted as an expert advisor and speaker for AbbVie and has received honoraria in this regard. He has also received research Grant funding from Astrozeneca. B. Fauroux has received compensation as a neonatology board member from Abbvie. J. Figueras-Aloy has acted as an expert advisor and speaker for AbbVie and has received honoraria in this regard. P. Manzoni has acted as a speaker for AbbVie, and as an expert advisor for AbbVie, TEVA, MedImmune, AstraZeneca, Janssen, and has received honoraria in this regard. B. Paes has received research funding from AbbVie Corporation and compensation as an advisor or lecturer from AbbVie and MedImmune. E.A.F Simões has received Grant funding to his institution from MedImmune Inc, Glaxo Smith Kline Inc, and received consultancy fees to the institution, from Abbvie. X. Carbonell-Estrany has acted as an expert advisor and speaker for AbbVie and has received honoraria in this regard.

Compliance with Ethics Guidelines. The analysis in this review article is based on previously published studies and does not involve any new studies of human subjects performed by any of the authors.

Open Access. This article is distributed under the terms of the Creative Commons Attribution-NonCommercial 4.0 International 
License (http://creativecommons.org/licenses/ by-nc/4.0/), which permits any noncommercial use, distribution, and reproduction in any medium, provided you give appropriate credit to the original author(s) and the source, provide a link to the Creative Commons license, and indicate if changes were made.

\section{REFERENCES}

1. Nair H, Nokes DJ, Gessner BD, Dherani M, Madhi SA, Singleton RJ, et al. Global burden of acute lower respiratory infections due to respiratory syncytial virus in young children: a systematic review and meta-analysis. Lancet. 2010;375:1545-55.

2. Haerskjold A, Kristensen K, Kamper-Jørgensen M, Andersen AMN, Ravn H, Stensballe LG. Risk factors for hospitalization for respiratory syncytial virus infection: a population-based cohort study of Danish children. Pediatr Infect Dis J. 2016;35:61-5.

3. Glezen WP, Taber LH, Frank AL, Kasel JA. Risk of primary infection and reinfection with respiratory syncytial virus. Am J Dis Child. 1986;140:543-6.

4. Gil-Prieto R, Gonzalez-Escalada A, Marín-García P, Gallardo-Pino C, Gil-de-Miguel A. Respiratory syncytial virus bronchiolitis in children up to 5 years of age in Spain: epidemiology and comorbidities: an observational study. Medicine (Baltimore). 2015;94:e831.

5. Simões EA, DeVincenzo JP, Boeckh M, Bont L, Crowe JE Jr, Griffiths P, et al. Challenges and opportunities in developing respiratory syncytial virus therapeutics. J Infect Dis. 2015;211(Suppl 1):S1-20.

6. Vicente D, Montes M, Cilla G, Perez-Yarza EG, Perez-Trallero E. Hospitalization for respiratory syncytial virus in the paediatric population in Spain. Epidemiol Infect. 2003;131:867-72.

7. Fjaerli HO, Farstad T, Bratlid D. Hospitalisations for respiratory syncytial virus bronchiolitis in Akershus, Norway, 1993-2000: a population-based retrospective study. BMC Pediatr. 2004;4:25.

8. Greenough A, Alexander J, Burgess S, Bytham J, Chetcuti PAJ, Hagan J, et al. Health care utilisation of prematurely born, preschool children related to hospitalisation for RSV infection. Arch Dis Child. 2004;89:673-8.
9. Paramore LC, Ciuryla V, Ciesla G, Liu L. Economic impact of respiratory syncytial virus-related illness in the US: an analysis of national databases. Pharmacoeconomics. 2004;22:275-84.

10. Horn SD, Smout RJ. Effect of prematurity on respiratory syncytial virus hospital resource use and outcomes. J Pediatrics. 2003;143(5 Suppl):S133-41.

11. Thorburn K. Pre-existing disease is associated with a significantly higher risk of death in severe respiratory syncytial virus infection. Arch Dis Child. 2009;94:99-103.

12. Hervás D, Reina J, Yañez A, del Valle JM, Figuerola J, Hervás JA. Epidemiology of hospitalization for acute bronchiolitis in children: differences between RSV and non-RSV bronchiolitis. Eur J Clin Microbiol Infect Dis. 2012;31:1975-81.

13. Murray J, Bottle A, Sharland M, Modi N, Aylin P, Majeed A, Saxena S, Medicines for Neonates Investigator Group. Risk factors for hospital admission with RSV bronchiolitis in England: a population-based birth cohort study. PLoS One. 2014;9:e89186.

14. Hall CB, Weinberg GA, Iwane MK, Blumkin AK, Edwards KM, Staat MA, et al. The burden of respiratory syncytial virus infection in young children. N Engl J Med. 2009;360:588-98.

15. Hall CB. The burgeoning burden of respiratory syncytial virus among children. Infect Disord Drug Targets. 2012;12:92-7.

16. Hall CB, Weinberg GA, Blumkin AK, Edwards KM, Staat MA, Schultz AF, et al. Respiratory syncytial virus-associated hospitalizations among children less than 24 months of age. Pediatrics. 2013;132:e341-8.

17. Broadbent L, Groves H, Shields MD, Power UF. Respiratory syncytial virus, an ongoing medical dilemma: an expert commentary on respiratory syncytial virus prophylactic and therapeutic pharmaceuticals currently in clinical trials. Influenza Other Respir Viruses. 2015;9:169-78.

18. World Health Organization. Research needs for the Battle against Respiratory Viruses (BRaVe). Background document 2013. http://www.who.int/influenza/ patient_care/clinical/BRaVe_Research_Agenda_2013. pdf?ua=1. Accessed 6 Apr 2016.

19. The IMpact-RSV Study Group. Palivizumab, a humanized respiratory syncytial virus monoclonal antibody, reduces hospitalization from respiratory syncytial virus infection in high-risk infants. Pediatrics. 1998;102:531-7. 
20. Committee on Infectious Diseases and Bronchiolitis Guidelines Committee. Updated guidance for palivizumab prophylaxis among infants and young children at increased risk of hospitalization for respiratory syncytial virus infection. Pediatrics. 2014;134:415-20.

21. Modjarrad K, Giersing B, Kaslow DC, Smith PG, Moorthy VS, WHO RSV Vaccine Consultation Expert Group. WHO consultation on respiratory syncytial virus vaccine development report from a World Health Organization Meeting held on 23-24 March 2015. Vaccine. 2016;4(34):190-7.

22. OCEBM Levels of Evidence Working Group. The Oxford 2011 levels of evidence. Oxford Centre for Evidence-Based Medicine. http://www.cebm.net/ index.aspx?o=5653. Accessed Aug 2015.

23. OCEBM Levels of Evidence Working Group. The Oxford 2009 Levels of Evidence. Oxford Centre for Evidence-Based Medicine http://www.cebm. net/oxford-centre-evidence-based-medicine-levelsevidence-march-2009/. Accessed Jan 2016.

24. Jadad AR, Moore RA, Carroll D, et al. Assessing the quality of reports of randomized clinical trials: is blinding necessary? Control Clin Trials. 1996;17:1-12.

25. Viswanathan M, Berkman ND, Dryden DM, Hartling L. Assessing risk of bias and confounding in observational studies of interventions or exposures: further development of the RTI Item Bank. Methods Research Report. (Prepared by RTI-UNC Evidence-based Practice Center under Contract No. 290-2007-10056-I). AHRQ Publication No. 13-EHC106-EF. Rockville, MD: Agency for Healthcare Research and Quality. 2013. http:// www.effectivehealthcare.ahrq.gov/reports/final.cfm. Accessed Jan 2016.

26. Higgins JPT, Altman DG (editors). Chapter 8: assessing risk of bias in included studies. In: Higgins JPT, Gree S, editors. Cochrane handbook for systematic reviews of interventions. Version 5.0.1 [updated September 2008]. The Cochrane Collaboration 2008. http://www.cochranehandbook.org. Accessed Jan 2016.

27. Constantopoulos AG, Kafetzis DA, Syrogiannopoulos GA, Roilides EJ, Malaka-Zafiriu EE, Sbyrakis SS, et al. Burden of respiratory syncytial viral infections on paediatric hospitals: a two-year prospective epidemiological study. Eur J Clin Microbiol Infect Dis. 2002;21:102-7.

28. Salvador García C, Moreno Docóna A, Piñero JA, Alfayate Miguelez S, Iborra Bendicho MA. Aetiology of bronchiolitis in hospitalised children in South-East Spain. An Pediatr (Barc). 2012;77:386-90.
29. Weigl JA, Puppe W, Schmitt HJ. Incidence of respiratory syncytial virus-positive hospitalizations in Germany. Eur J Clin Microbiol Infect Dis. 2001;20:452-9.

30. Zuccotti G, Dilillo D, Zappa A, Galli E, Amendola A, Martinelli $\mathrm{M}$, et al. Epidemiological and clinical features of respiratory viral infections in hospitalized children during the circulation of influenza virus $\mathrm{A}(\mathrm{H} 1 \mathrm{~N} 1)$ 2009. Influenza Other Respir Viruses. 2011;5:e528-34.

31. Frassanito A, Nicolai A, Nenna R, Cangiano G, Papasso S, Mancino E, et al. Acute viral respiratory tract infections and wheezing at follow-up in children <3 years. Eur Respir J. 2015;46(Suppl 59):PA1319.

32. Ferrara M, Cangiano G, Papasso S, Nicolai A, Evangelisti M, Nenna R, et al. Bronchiolitis: a 9 years epidemic seasons analysis. Eur Respir J. 2014;44(Suppl 58):P1258.

33. Lanari M, Giovannini M, Giuffré L, Marini A, Rondini G, Rossi GE, the Investigators R.A.DA.R. Study Group, et al. Prevalence of respiratory syncytial virus infection in Italian infants hospitalized for acute lower respiratory tract infections, and association between respiratory syncytial virus infection risk factors and disease severity. Pediatr Pulmonol. 2002;33:458-65.

34. Müller-Pebody B, Edmunds WJ, Zambon MC, Gay NJ, Crowcroft NS. Contribution of RSV to bronchiolitis and pneumonia-associated hospitalizations in English children, April 1995-March 1998. Epidemiol Infect. 2002;129:99-106.

35. Stockman LJ, Curns AT, Anderson LJ, Fischer-Langley G. Respiratory syncytial virus-associated hospitalizations among infants and young children in the United States, 1997-2006. Pediatr Infect Dis J. 2012;31:5-9.

36. Santibanez P, Gooch K, Vo P, Lorimer M, Sandino Y. Acute care utilization due to hospitalizations for pediatric lower respiratory tract infections in British Columbia, Canada. BMC Health Serv Res. 2012;12:451.

37. Ajayi-Obe EK, Coen PG, Handa R, Hawrami K, Aitken $\mathrm{C}$, McIntosh EDG, et al. Influenza A and respiratory syncytial virus hospital burden in young children in East London. Epidemiol Infect. 2008;136:1046-58.

38. Corsello G, Di Carlo P, Salsa L, Gabriele B, Meli L, Bruno $S$, et al. Respiratory syncytial virus infection in a Sicilian pediatric population: risk factors, epidemiology, and severity. Allergy Asthma Proc. 2008;29:205-10. 
39. Calvo C, Pozo F, García-García ML, Sanchez M, Lopez-Valero M, Pérez-Breña P. Detection of new respiratory viruses in hospitalized infants with bronchiolitis: a three-year prospective study. Acta Paediatr. 2010;99:883-7.

40. Díez Domingo J, Ridao López M, Ubeda Sansano I, Ballester Sanz A. Incidence and cost of hospitalizations for bronchiolitis and respiratory syncytial virus infections in the autonomous community of Valencia in Spain (2001 and 2002). An Pediatr (Barc). 2006;65:325-30.

41. García García ML, Ordobás Gabin M, Calvo Reya C, González Alvarez M, Aguilar Ruiz J, Arregui Sierra A, et al. Viral infection of the lower respiratory tract in hospitalized infants: etiology, clinical features and risk factors. An Esp Pediatr. 2001;55:101-7.

42. García-García ML, Calvo C, Pozo F, Villadangos PA, Pérez-Breña $\mathrm{P}$, Casas I. Spectrum of respiratory viruses in children with community-acquired pneumonia. Pediatr Infect Dis J. 2012;31:808-13.

43. Artiles-Campelo F, Pérez-González MC, Caballero-Hidalgo A, Pena-López MJ. Etiology of acute viral respiratory tract infections in children from Gran Canaria, the Canary Islands (Spain). Enferm Infec Microbiol Clin. 2006;24:556-61.

44. García CG, Bhore R, Soriano-Fallas A, Trost M, Chason R, Ramilo O, et al. Risk factors in children hospitalized with RSV bronchiolitis versus non-RSV bronchiolitis. Pediatrics. 2010;126:e1453-60.

45. Iwane MK, Edwards KM, Szilagyi PG, Walker FJ, Griffin MR, Weinberg GA, New Vaccine Surveillance Network, et al. Population-based surveillance for hospitalizations associated with respiratory syncytial virus, influenza virus, and parainfluenza viruses among young children. Pediatrics. 2004;113:1758-64.

46. Mlinaric-Galinovic G, Vilibic-Cavlek T, Ljubin-Sternak S, Drazenovic V, Galinovic I, Tomic $\mathrm{V}$, et al. Eleven consecutive years of respiratory syncytial virus outbreaks in Croatia. Pediatr Int. 2009;51:237-40.

47. Gooskens J, van der Ploeg V, Sukhai RN, Vossen ACTM, Eric CJ, Claas ECJ, et al. Clinical evaluation of viral acute respiratory tract infections in children presenting to the emergency department of a tertiary referral hospital in the Netherlands. BMC Pediatr. 2014;14:297.

48. El-Hajje MJ, Lambe C, Moulin F, de Souremain N, Pons-Catalano C, Chalumeau M, et al. The burden of respiratory viral disease in hospitalized children in Paris. Eur J Pediatr. 2008;167:435-6.
49. Zhou H, Thompson WW, Viboud CG, Ringholz CM, Cheng P-Y, Steiner C, et al. Hospitalizations associated with influenza and respiratory syncytial virus in the United States, 1993-2008. Clin Infect Dis. 2012;54:1427-36.

50. Medici MC, Arcangeletti MC, Rossi GA, Lanari M, Merollo R, Paparatti UD, Osservatorio VRS Study Group, et al. Four year incidence of respiratory syncytial virus infection in infants and young children referred to emergency departments for lower respiratory tract diseases in Italy: the "Osservatorio VRS" Study (2000-2004). New Microbiol. 2006;29:35-43.

51. Zomer-Kooijker K, Uiterwaal CS, van der Gugten AC, Wilbrink B, Bont LJ, van der Ent CK. Decreased lung function precedes severe respiratory syncytial virus infection and post-respiratory syncytial virus wheeze in term infants. Eur Respir J. 2014;44:666-74.

52. Tatochenko V, Uchaikin V, Gorelov A, Gudkov K, Campbell A, Schulz G, et al. Epidemiology of respiratory syncytial virus in children $\leq 2$ years of age hospitalized with lower respiratory tract infections in the Russian Federation: a prospective, multicenter study. Clin Epidemiol. 2010;21(2):221-7.

53. Bicer S, Giray T, Çöl D, Çiler Erdă̆ G, Vitrinel A, Yesim Gürol $Y$, et al. Virological and clinical characterizations of respiratory infections in hospitalized children. Ital J Pediatr. 2013;39:22.

54. Hacımustafaoğlu M, Celebi S, Bozdemir SE, Ozgür T, Ozcan I, Güray A, et al. RSV frequency in children below 2 years hospitalized for lower respiratory tract infections. Turk J Pediatr. 2013;55:130-9.

55. Turkish Neonatal Society. The seasonal variations of respiratory syncytial virus infections in Turkey: a 2-year epidemiological study. Turk J Pediatr. 2012;54:216-22.

56. Resch B, Gusenleitner W, Mandl C, Müller W. Epidemiology of respiratory syncytial virus infection in Southern Austria. Pediatr Infect Dis J. 2000;19:587-8.

57. Resch B, Gusenleitner W, Müller W. The impact of respiratory syncytial virus infection: a prospective study in hospitalized infants younger than 2 years. Infection. 2002;30:193-7.

58. Boyce TG, Mellen BG, Mitchel EF Jr, Wright PF, Griffin MR. Rates of hospitalization for respiratory syncytial virus infection among children in medicaid. J Pediatr. 2000;137:865-70.

59. Fleming DM, Pannell RS, Elliot AJ, Cross KW. Respiratory illness associated with influenza and 
respiratory syncytial virus infection. Arch Dis Child. 2005;90:741-6.

60. Bourgeois FT, Valim C, McAdam AJ, Mandl KD. Relative impact of influenza and respiratory syncytial virus in young children. Pediatrics. 2009;124:e1072-80.

61. Svensson C, Berg K, Sigurs N, Trollfors B. Incidence, risk factors and hospital burden in children under five years of age hospitalised with respiratory syncytial virus infections. Acta Paediatr. 2015;104:922-6.

62. Leader S, Kohlhase K. Recent trends in severe respiratory syncytial virus (RSV) among US infants, 1997 to 2000. J Pediatr. 2003;143:S127-32.

63. Deshpande SA, Northern V. The clinical and health economic burden of respiratory syncytial virus disease among children under 2 years of age in a defined geographical area. Arch Dis Child. 2003;88:1065-9.

64. Grimaldi M, Cornet B, Milou C, Gouyon JB. Prospective regional study of an epidemic of respiratory syncytial virus (RSV) bronchiolitis. Arch Pediatr. 2002;9:572-80.

65. Johnson JI, Ratard R. Respiratory syncytial virus-associated hospitalizations in Louisiana. J La State Med Soc. 2012;164:268-73.

66. Liese JG, Grill E, Fischer B, Roeckl-Wiedmann I, Carr $\mathrm{D}$, Belohradsky $\mathrm{BH}$. Incidence and risk factors of respiratory syncytial virus-related hospitalizations in premature infants in Germany. Eur J Pediatr. 2003;162:230-6.

67. McCormick J, Tubman R. Readmission with respiratory syncytial virus (RSV) infection among graduates from a neonatal intensive care unit. Pediatr Pulmonol. 2002;34:262-6.

68. Shay DK, Holman RC, Newman RD, Liu LL, Stout JW, Anderson LJ. Bronchiolitis-associated hospitalizations among US children, 1980-1996. JAMA. 1999;282:1440-6.

69. Langley JM, LeBlanc JC, Smith B, Wang EEL. Increasing incidence of hospitalization for bronchiolitis among Canadian children, 1980-2000. J Infect Dis. 2003;188:1764-7.

70. Hasegawa K, Tsugawa Y, Brown DF, Mansbach JM, Camargo CA Jr. Trends in bronchiolitis hospitalizations in the United States, 2000-2009. Pediatrics. 2013;132:28-36.

71. Fournel I, Soulias M, Bour JB, Gouyon JB, Huet F, Aho LS. Evolution of the number of rotavirus and respiratory syncytial virus infections in children hospitalised in a French university hospital between 1998 and 2005. Pathol Biol (Paris). 2010;58:406-14.

72. Ortiz de Lejarazu R, Rojo S, Bermejo-Martín JF, Almansa R, Solis P, Tamames S, et al. Trend and seasonality of respiratory syncytial virus in Valladolid (Spain) during 1993-2010. Acta Pediatr Esp. 2012;70:1-8.

73. Centers for Disease Control and Prevention (CDC). Respiratory syncytial virus-United States, July 2007-June 2011. MMWR Morb Mortal Wkly Rep. 2011;60:1203-6.

74. Centers for Disease Control and Prevention (CDC). Respiratory syncytial virus activity-United States, July 2011-January 2013. MMWR Morb Mortal Wkly Rep. 2013;62:141-4.

75. Alonso A, Andres JM, Garmendia JR, Diez I, Gil JM, Ardura J. Bronchiolitis due to respiratory syncytial virus in hospitalized children: a study of seasonal rhythm. Acta Paediatr. 2007;96:731-5.

76. Calvo C, García-García ML, Pozo F, Paula G, Molinero $\mathrm{M}$, Caldéron $\mathrm{A}$, et al. Respiratory syncytial virus coinfections with rhinovirus and human bocavirus in hospitalized children. Medicine (Baltimore). 2015;94:e1788.

77. Tanner H, Boxall E, Osman H. Respiratory viral infections during the 2009-2010 winter season in Central England, UK: incidence and patterns of multiple virus coinfections. Eur J Clin Microbiol Infect Dis. 2012;3:3001-6.

78. Thorburn K, Harigopal S, Reddy V, Taylor N, van Saene HKF. High incidence of pulmonary bacterial co-infection in children with severe respiratory syncytial virus (RSV) bronchiolitis. Thorax. 2006;61:611-5.

79. Weigl JA, Puppe W, Rockahr S, Schmitt HJ. Burden of disease in hospitalized RSV-positive children in Germany. Klin Padiatr. 2002;214:334-42.

80. Martínez-Roig A, Salvadó M, Caballero-Rabasco MA, Sánchez-Buenavida A, López-Segura N, Bonet-Alcaina M. Viral coinfection in childhood respiratory tract infections. Arch Bronconeumol. 2015;51:5-9.

81. Calvo C, García-García ML, Blanco C, Vázquez MC, Frías ME, Pérez-Breña $P$, et al. Multiple simultaneous viral infections in infants with acute respiratory tract infections in Spain. J Clin Virol. 2008;42:268-72.

82. Asner SA, Rose W, Petrich A, Richardson S, Tran DJ. Is virus coinfection a predictor of severity in children with viral respiratory infections? Clin Microbiol Infect. 2015;21:264.e1-6. 
83. Kristensen K, Dahm T, Frederiksen PS, Ibsen J, Iyore E, Mller Jensen E, et al. Epidemiology of respiratory syncytial virus infection requiring hospitalization in East Denmark. Pediatr Infect Dis J. 1998;17:996-1000.

84. Tsolia MN, Kafetzis D, Danelatou K, Astra H, Kallergi $\mathrm{K}$, Spyridis P, et al. Epidemiology of respiratory syncytial virus bronchiolitis in hospitalized infants in Greece. Eur J Epidemiol. 2003;18:55-61.

85. Flores P, Rebelo-de-Andrade H, Gonçalves $\mathrm{P}$, Guiomar R, Carvalho C, Sousa EN, et al. Bronchiolitis caused by respiratory syncytial virus in an area of Portugal: epidemiology, clinical features, and risk factors. Eur J Clin Microbiol Infect Dis. 2004;23:39-45.

86. Eriksson M, Bennet R, Rotzén-Ostlund $M$, von Sydow M, Wirgart BZ. Population-based rates of severe respiratory syncytial virus infection in children with and without risk factors, and outcome in a tertiary care setting. Acta Paediatr. 2002;91:593-8.

87. Brooks AM, McBride JT, McConnochie KM, Aviram M, Long C, Hall CB. Predicting deterioration in previously healthy infants hospitalized with respiratory syncytial virus infection. Pediatrics. 1999;104:463-7.

88. Gijtenbeek RG, Kerstjens JM, Reijneveld SA, Duiverman EJ, Bos AF, Vrijlandt EJLE. RSV infection among children born moderately preterm in a community-based cohort. Eur J Pediatr. 2015;174:435-42.

89. Brandenburg AH, Jeannet P-Y, Steensel-Moll HAV, Ott A, Rothbarth PH, Wunderli W, et al. Local variability in respiratory syncytial virus disease severity. Arch Dis Child. 1997;77:410-4.

90. Wang EE, Law BJ, Stephens D. Pediatric Investigators Collaborative Network on Infections in Canada (PICNIC) prospective study of risk factors and outcomes in patients hospitalized with respiratory syncytial viral lower respiratory tract infection. J Pediatr. 1995;126:212-9.

91. Soilly AL, Ferdynus C, Desplanches O, Grimaldi M, Gouyon JB. Paediatric intensive care admissions for respiratory syncytial virus bronchiolitis in France: results of a retrospective survey and evaluation of the validity of a medical information system programme. Epidemiol Infect. 2012;140:608-16.

92. Berger A, Obwegeser E, Aberle SW, Langgartner M, Popow-Kraupp T. Nosocomial transmission of respiratory syncytial virus in neonatal intensive care and intermediate care units: a prospective epidemiologic study. Pediatr Infect Dis J. 2010;29:669-70.
93. Simon A, Müller A, Khurana K, Engelhart S, Exner M, Schildgen O, DSM RSV Paed Study Group, et al. Nosocomial infection: a risk factor for a complicated course in children with respiratory syncytial virus infection-results from a prospective multicenter German surveillance. Int J Hyg Environ Health. 2008;211(3-4):241-50.

94. Alan S, Erdeve O, Cakir U, Akduman H, Zenciroglu A, Akcakus M, et al. Outcome of the Respiratory Syncytial Virus related acute lower respiratory tract infection among hospitalized newborns: a prospective multicenter study. J Matern Fetal Neonatal Med. 2015;12:1-8 (Epub ahead of print).

95. Lozano R, Naghavi M, Foreman K, Lim S, Shibuya K, Aboyans $\mathrm{V}$, et al. Global and regional mortality from 235 causes of death for 20 age groups in 1990 and 2010: a systematic analysis for the Global Burden of Disease Study 2010. Lancet. 2012;380:2095-128.

96. Thompson WW, Shay DK, Weintraub E, et al. Mortality associated with influenza and respiratory syncytial virus in the United States. JAMA. 2003;289:179-86.

97. Berkley JA, Munywoki P, Ngama M, et al. Viral etiology of severe pneumonia among Kenyan infants and children. JAMA. 2010;303:2051-7.

98. Nokes DJ, Ngama M, Bett A, et al. Incidence and severity of respiratory syncytial virus pneumonia in rural Kenyan children identified through hospital surveillance. Clin Infect Dis. 2009;49:1341-9.

99. Shay DK, Holman RC, Roosevelt GE, Clarke MJ, Andersen LJ. Bronchiolitis-associated mortality and estimates of respiratory syncytial virus-associated deaths among US children, 1979-1997. J Infect Dis. 2001;183:16-22.

100. Fryzek JP, Martone WJ, Groothuis JR. Trends in chronologic age and infant respiratory syncytial virus hospitalization: an 8-year cohort study. Adv Ther. 2011;28:195-201.

101. Bradley JP, Bacherier LB, Bonfiglio J, Schechtman KB, Strunk R, Storch G, et al. Severity of respiratory syncytial virus is affected by cigarette smoke exposure and atopy. Pediatrics. 2005;115:7-14.

102. von Linstow ML, Høgh M, Nordbø SA, Eugen-Olsen J, Koch A, Høgh B. A community study of clinical traits and risk factors for human metapneumovirus and respiratory syncytial virus infection during the first year of life. Eur J Pediatr. 2008;167:1125-33.

103. Schuurhof A, Bont L, Siezen CL, Hodemaekers H, van Houwelingen HC, Kimman TG, et al. Interleukin-9 polymorphism in infants with respiratory syncytial virus infection: an opposite 
effect in boys and girls. Pediatr Pulmonol. 2010;45:608-13.

104. Choudhuri JA, Ogden LG, Ruttenber AJ, Thomas DS, Todd JK, Simoes EA. Effect of altitude on hospitalizations for respiratory syncytial virus infection. Pediatrics. 2006;117:349-56.

105. Kristensen K, Fisker N, Haerskjold A, Henrik R, Simoes EAF, Stensballe L. Caesarean section and hospitalization for respiratory syncytial virus infection: a population-based study. Pediatr Infect Dis J. 2015;34:145-8.

106. Iwane MK, Chaves SS, Szilagyi PG, Edwards KM, Hall CB, Staat MA, et al. Disparities between black and white children in hospitalizations associated with acute respiratory illness and laboratory-confirmed influenza and respiratory syncytial virus in 3 US counties-2002-2009. Am J Epidemiol. 2013;177:656-65.

107. Sangaré L, Curtis MP, Ahmad S. Hospitalization for respiratory syncytial virus among California infants: disparities related to race, insurance, and geography. J Pediatr. 2006;149:373-7.

108. Nielsen HE, Siersma V, Andersen S, Gahrn-Hansen $\mathrm{B}$, Mordhorst $\mathrm{CH}$, Nørgaard-Pedersen B, et al. Respiratory syncytial virus infection-risk factors for hospital admission: a case-control study. Acta Paediatr. 2003;92:1314-21.

109. Rossi GA, Medici MC, Arcangeletti MC, Lanari M, Merolla R, Paparatti U, et al. Risk factors for severe $\mathrm{RSV}$-induced lower respiratory tract infection over four consecutive epidemics. Eur J Pediatr. 2007;166:1267-72.

110. Cilla G, Sarasua A, Montes M, Arostegui N, Vicente $D$, Pérez-Yarza E, et al. Risk factors for hospitalization due to respiratory syncytial virus infection among infants in the Basque Country, Spain. Epidemiol Infect. 2006;134:506-13.
111. Belderbos ME, Houben ML, Wilbrink B, Lentjes E, Bloemen EM, Kimpen JL, et al. Cord blood vitamin D deficiency is associated with respiratory syncytial virus bronchiolitis. Pediatrics. 2011;127:e1513-20.

112. Stensballe LG, Kristensen K, Simoes EA, Jensen H, Nielsen J, Benn CS, et al. Atopic disposition, wheezing, and subsequent respiratory syncytial virus hospitalization in Danish children younger than 18 months: a nested case-control study. Pediatrics. 2006;118:e1360-8.

113. Trefny P, Stricker T, Baerlocher C, Sennhauser FH. Family history of atopy and clinical course of RSV infection in ambulatory and hospitalized infants. Pediatr Pulmonol. 2000;30:302-6.

114. Lapeña S, Robles MB, Castañón L, Martínez JP, Reguero S, Alonso MP, et al. Climatic factors and lower respiratory tract infection due to respiratory syncytial virus in hospitalised infants in northern Spain. Eur J Epidemiol. 2005;20:271-6.

115. Vandini S, Corvaglia L, Alessandroni R, Aquilano G, Marsico C, Spinelli M, et al. Respiratory syncytial virus infection in infants and correlation with meteorological factors and air pollutants. Ital J Pediatr. 2013;39:1.

116. Karr CJ, Rudra CB, Miller KA, Gould TR, Larson T, Sathyanarayana $S$, et al. Infant exposure to fine particulate matter and traffic and risk of hospitalization for RSV bronchiolitis in a region with lower ambient air pollution. Environ Res. 2009;109:321-7.

117. Jansson L, Nilsson P, Olsson M. Socioeconomic environmental factors and hospitalization for acute bronchiolitis during infancy. Acta Paediatr. 2002;91:335-8. 